\title{
Role of openness in industrial internet platform providers' strategy
}

\author{
Karan Menon ${ }^{1}$, Hannu Kärkkäinen ${ }^{1}$, and Thorsten Wuest ${ }^{2}$ \\ 1 Tampere University of Technology, Finland, \\ karan.menon@tut.fi, hannu.karkkainen@tut.fi \\ 2 West Virginia University, USA, \\ thwuest@mail. wvu.edu
}

\begin{abstract}
Industrial internet, Industry 4.0 and Cyber-Physical Systems (CPS) can be collectively defined as industrial systems that integrate computational and physical capabilities of machines in order to provide advanced analytics and interact with humans. Industrial internet platforms allow the industrial companies to manage data, information and knowledge effectively within and between product lifecyle phases. Industrial internet platform's openness plays a very important role in decision making related to platform selection for industrial companies. This paper focuses on various dimensions of openness and how it effects the strategy of platform owners or providers and how this strategy effects in short and long term to their end-users. In order to analyze the above Kaa IoT and PTC ThingWorx have been analyzed to understand the impact of openness dimensions on their strategy and business.
\end{abstract}

Keywords: Industrial Internet, Platforms, PLM, Openness

\section{Introduction}

Industrial internet, Industry 4.0 and Cyber-Physical Systems (CPS) can be collectively defined as industrial systems that integrate computational and physical capabilities of machines in order to provide advanced analytics and interact with humans [1-4]. Industrial internet, or Industrial Internet of Things (IIoT) will go way beyond the traditional factory automation: the related applications will change the structure of various industries and even the structure and foundations of their competition (e.g. [5]), and can for e.g. significantly reduce the transaction costs for various industrial transactions in the value chain. It has also been found that industrial internet and the related rather lately matured technologies (e.g. sensors, data analytics and various types of industrial internet platforms) can bring significant benefits and possibilities to the management of product lifecycle information, for instance joining various types of data, information and knowledge from different lifecycle phases in a more efficient manner, and facilitating the so-called Closed-Loop PLM (e.g. [6]).

The significance of various types of platforms has grown increasingly in various industries (e.g. [7]) as well as in industrial internet (e.g. [8]). Importantly, 
platforms and platform-like digital services can provide new ways to access and accelerate the capturing of data and converting it into insightful information and knowledge. Access to data, information and knowledge across the life cycle phases and within the different phases is the key to the value creation from product related lifecycle data, information and knowledge [9]. Platform openness has been an interesting topic both academically and for companies - openness has been found to be one of the key concepts in the design and governance of platform ecosystems (e.g. [10]). There are various strategies related to openness of platforms, and platform openness can provide both significant benefits and risks or disadvantages for both platform providers and platform users alike. For instance, according to [11], opening a platform can increase platform adoption by network effects, reduce users' concerns about lock-in, stimulate the creation of differentiated goods that meet better the user needs, as well as reduce users' switching costs and increase platform provider competition.

Currently, there are significant differences in the degree of platforms' openness, i.e. how 'open' the platforms are for example in letting third party developers and companies to make applications over the platform using the data and information from the platform $[7,11]$. The benefits of various types of platform openness, especially the long-term ones, as well as the potential downsides of openness, are often difficult to be understood, and thus, the selection of most suitable platforms can be challenging for platform users. Furthermore, according to a recent review [10], openness has been mostly discussed from a technologyoriented perspective, while openness is closely related to how access is granted to technology. Finally, the long-term benefits and downsides of platform openness have been very little studied in the more specific context of industrial internet, and we have not been able to find related studies especially from the perspective of industrial internet platform users.

Accordingly, we have derived the following research questions to address the existing research gaps related to platform openness, as well as its importance to platform users especially in the context of industrial internet platforms:

1. What is the current status and future plans of the industrial internet platforms towards openness with respect to different types of platform openness?

2. What is the overall strategy regarding platform openness for the industrial internet platform providers?

3. What kinds of short and long-term impacts (Benefits and Risks) of the different types of platform providers' openness strategy are there for the industrial internet platform users?

The rest of the paper is divided into theoretical background, research methodology and design, results and findings, discussion and conclusions.

\section{Theoretical Background}

\subsection{Industrial Internet Platforms}

Platforms on a very broad level can be divided into 'internal' or firm level platforms and 'external' or ecosystem level (industry wide) platforms. We follow the 
definition of Industry Platform by [7]. According to them, "industry platforms are defined as products, services, or technologies developed by one or more firms, that serve as foundations upon which a larger number of firms can build further complementary innovations and potentially generate network effects." External or industry platforms are probably the most relevant forms of platforms in the context of PLM, because they can enhance the management of data, information and knowledge not only internally, but also amongst the various organizational actors (stakeholders) throughout the lifecycle phases (BOL, MOL, EOL). In case of industry or external platforms, there are differences in the degree of platforms' openness meaning how 'open' the platform is in order to let third party developers and companies while develop applications for the platform using the data and information from the platform $[7,11]$.

Industrial Internet, Industry 4.0 and CPS can be collectively defined as industrial systems that integrate computational and physical capabilities of machines in order to provide advanced analytics and interactions with humans ([12, 1,2,4, 3].Industrial internet platforms can access data from different sensors, actuators, enterprise systems, social media and other novel data sources $[13,14]$. The industrial internet platform is able to aggregate data into a single database which can be stored, either in dedicated in-house servers or with other third party cloud storage providers $[2,3]$.

In the context of PLM, there has been a marked shift in its vision, which would ideally mean the ability to access, manage and control product related information across all phases of the lifecycle [15]. In case of PLM, industrial internet platforms can provide the real time management of data and information

flows as well as help in the data-information-knowledge (D-I-K) transformations along all phases of the product lifecycle.

\subsection{Industrial Internet Platforms' Openness and PLM}

Industrial companies need to select the platforms based on optimal levels of openness because of their requirement to use the platforms with various different actors (for example: suppliers, customers, designers). Furthermore, in PLM context industrial internet platform openness can provide variety of benefits, possibilities and restrictions considering the management of D-I-K both within and between lifecycle phases. As defined by Eisenmann et al. 2009 [18], A platform is "open", as long as, 1) no restrictions are placed on participation in its development, commercialization or use; or 2) any restrictions-for example, requirements to conform with technical standards or pay licensing gees-are reasonable and non-discriminatory, that is, they are applies to all the potential platform participants. This definition is applicable to variety of actors that participate in the creation, usage and propagation of the platform. These actors are distributed in four categories; 1) demand-side platform users, basically endusers 2) supply-side platform users, complementors or 3rd party developers 3 ) platform providers 4) platform sponsors.

In this study we have used the analytical framework presenting the dimensions of platform openness and their detailed sub-dimensions developed in our 
Table 1. Dimensions and sub-dimensions of platform openness [16]

\begin{tabular}{|c|c|c|}
\hline Dimensions of Openness & $\begin{array}{l}\text { Detailed Sub-Dimensions } \\
\text { of Openness }\end{array}$ & Definitions \\
\hline \multirow{3}{*}{$\begin{array}{l}\text { Demand-Side User } \\
\text { (End User) }\end{array}$} & Access to information & $\begin{array}{l}\text { Level of access to information on } \\
\text { interfaces to link to the platform } \\
\text { or utilize its capabilities [7]. }\end{array}$ \\
\hline & Cost of access & $\begin{array}{l}\text { Cost of access as in patent or } \\
\text { licensing fees[7]. }\end{array}$ \\
\hline & $\begin{array}{l}\text { Control in terms of rules } \\
\text { to use the platform }\end{array}$ & $\begin{array}{l}\text { Types of rules governing use of } \\
\text { the platform[7]. }\end{array}$ \\
\hline \multirow[t]{3}{*}{$\begin{array}{l}\text { Supply-side User } \\
\text { (Application } \\
\text { Developer) }\end{array}$} & Core Developers & $\begin{array}{l}\text { They are developers employed } \\
\text { by the platform management } \\
\text { company itself. They develop tools } \\
\text { and applications which allows the } \\
\text { users to use the platform effectively[17]. }\end{array}$ \\
\hline & Extension Developers & $\begin{array}{l}\text { They are outside parties or 3rd party } \\
\text { developers who add features } \\
\text { (applications) and value to the } \\
\text { platform to enhance the functionality } \\
\text { of the platform[17]. }\end{array}$ \\
\hline & Data Aggregators & $\begin{array}{l}\text { Data aggregators collect different } \\
\text { interaction based data and re-sell } \\
\text { it to the companies (as per the platform } \\
\text { laws), who then can target } \\
\text { advertisements etc to the users[17]. }\end{array}$ \\
\hline \multirow{4}{*}{$\begin{array}{l}\text { Platform Provider } \\
\text { and Sponsor related } \\
\text { openness }\end{array}$} & Proprietary Model & $\begin{array}{l}\text { A single firm plays both provider and } \\
\text { sponsor role[18]. }\end{array}$ \\
\hline & Licensing Model & $\begin{array}{l}\text { A single firm sponsors the platform } \\
\text { then licences to multiple providers[18]. }\end{array}$ \\
\hline & Joint Venture Model & $\begin{array}{l}\text { Multiple sponsors jointly sponsor } \\
\text { the platform but a single firm serves as } \\
\text { its sole provider[18]. }\end{array}$ \\
\hline & Shared Model & $\begin{array}{l}\text { Multiple sponsors collaborate to develop } \\
\text { the platform's technology and then } \\
\text { compete with each other to provide } \\
\text { differentiated but compatible versions } \\
\text { to the users[18]. }\end{array}$ \\
\hline
\end{tabular}

previous study[16]. The conceptual analytical frame work can be found in Table 1.

Openness within lifecycle phases In this paragraph the value and characteristic of openness within the different lifecycle phases (BOL, MOL and EOL) is discussed. The general structure is based on the three main 'openness criteria' identified previously (see table 1).

The openness from demand-side (end-user) can generally be understood to be essential during the BOL and EOL phase and in most cases also during the MOL phase. During BOL and MOL, the end users are relatively clearly defined and their openness requirements are also well understood. When investigating the openness requirement from the supplier-side (application developer), the different phases are more homogeneous than the previously discussed demand-side. In terms of the openness criteria platform provider and platform sponsor, the implications on the different lifecycle phases are mostly related to interoperability issues.

Openness between the lifecycle phases The following structure presents a simplified view on the interfaces of the three main lifecycle phases. There might 


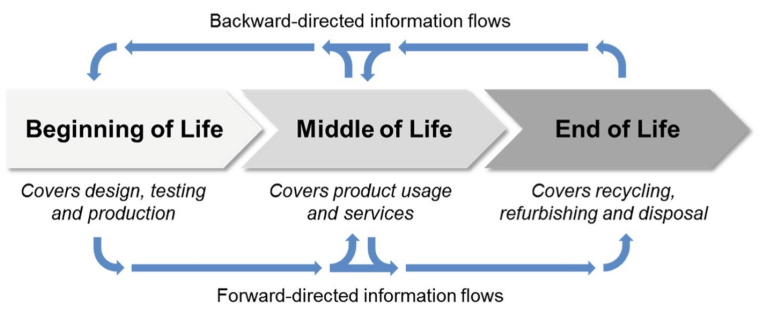

Fig. 1. Product lifecycle management phases and information flows within and between phases [19]

be more complex constellations that require taking all phases in a more networked structure into account to replicate interdependencies between all phases. However, this needs to be studied in detail and is not in the focus of this manuscript. More information regarding the information flows between different phases themselves can be found in[20,19]

The demand side (end user) openness requirements at the interface between BOL - MOL are expected to be high. Information access over lifecycle phase borders is essential for many applications. A rather common application of such cross-border information exchange that demands openness is design based on usage data[21]. The same high requirements towards openness stand true for BOL - MOL and MOL - EOL interfaces. From the supplier side (application developer) openness criteria, the interfaces are rather important as well. Designing an application to collect usage data for use during the beginning of live requires a high degree of openness regarding the interface between BOL - MOL for example. And this certainly stands true for other cross platform applications. With regard to the openness criteria platform provider and platform sponsor, the same arguments can be used for the interfaces between phases as for the phases themselves.

\subsection{Short and long term benefits and risks}

When an industrial company selects a particular industrial internet platform, it has to take into account various benefits and risks, both short and long term ones. In this study we focus specifically on the short and long term benefits and risks of platform openness from the viewpoint of the end-user or customer. There are a few studies that discuss the long and short term benefits and risks of Internet of Things (IoT) or Industrial internet platforms. The immediate or short term benefits, such as the fastened implementation of industrial internet or industry 4.0 for the company, fast access to various types of data, information and knowledge, aggregation of various types of different data into a single database for analytics, and potential at least short-term cost savings (provided that the platform purchasing and/or usage is free or nearly free) and others have been discussed in the literature $[2,3,8]$. Long term benefits, such as interoperability 
between companies and different software at a large scale, facilitating the "closedloop PLM" (facilitating the linking of data, information and knowledge between and within the lifecycle phases), and stimulation of the creation of differentiated goods to better user needs, have been noted as important, as well $[11,16]$. Short term downsides and risks, such as security risks, heavy investments play an important role while selecting a platform. Long term risks are not that easy to point out because the technology is so new, but still risks like lockin and security risks are very important to take into consideration. However, as a summary, the aforementioned papers on benefits and risks of industrial internet platforms are largely non-empirical and conceptual, and actual empirical studies in the recognition and analyses of industrial internet platforms' short and long-term benefits and risks, especially the benefits and risks of platform openness from the viewpoint of end-users and customers are lacking.

\section{Research Methodology and Design}

In this article we focus on impact of openness and related dimensions on industrial internet platforms. The underlying idea of platform openness and its effect on the strategy of platform companies is assessed using the framework developed in our previous papers [16]. Qualitative approach is used in this research because of the novelty of industrial internet platforms and related openness [16]. In this article the interest is to understand the strategy related to platform openness that creates long term and short term benefits and risks for user companies from the platform company's perspective. "Why?" questions offer a deeper understanding and explanation of strategies selected by platform companies. The qualitative approach has different underlying methods for collecting primary data [22], including interviews, group discussions, ethnography, participating observations, and experiments. We use semi-structured interviews to gain detailed insights because they allow a structured approach while leaving room for in-depth analysis. In the questionnaire, there are open and closed, simple and complex, and direct and indirect questions. The questions were created based on the findings in the theoretical section and with regard to the interviewee type [22]. The basic structure of the questionnaire is discussed in section 3.1 in detail.

The selection of the interview partners was undertaken under the premise of selecting cases which are typical for the process. In this article, we selected cases which had maximum variation between them but also were representatives of major industrial internet platforms. Kaa-IoT is a completely open source platform with limited personnel managing the platform's development and maintenance. PTC ThingWorx is a one of the most popular and one of the largest revenue generating (100 Million USD in 2016 fiscal year ${ }^{1}$ ) industrial internet platforms. If platform openness was put in a continuum then Kaa IoT would be at one end of the continuum (almost completely open) and PTC ThingWorx would be closer to the other end of the continuum. Hence, discussing their strategic views on openness provides a large variation to the research. All the interviewees are

\footnotetext{
${ }^{1}$ http://www.ptc.com/news/2016/ptc-announces-q4-fy16-results
} 
in high positions in their respective platforms, with the position of CEO, CTO or something similar. All interviews, ranging from 45 minutes to one hour, were carried out via phone, Skype, or in a face-to-face meeting. The qualitative interviews were conducted in the beginning of 2017. The interviews were recorded, hence data was prepared by transcribing the recordings. The answers were analyzed by their type and depth to identify the underlying meaning.

\subsection{Components of the questionnaire}

The qualitative semi-structured interview was basically divided into the following components: Strategy component, Openness dimensions related component, PLM component, Long term and short term benefits and risks component

Strategy component Questions in this particular component address the overall strategy of the industrial internet platform related to openness. Long term and short term strategies were also discussed in this component.

Openness dimensions related component This component addresses questions related to the strategies for openness dimensions and sub-dimensions in Table 1.

PLM component This component brings out the information regarding the respective platform's strategies when it comes to sharing data, information and knowledge within and in between lifecycle phases. Section 2.2 points towards the impact of openness in industrial internet platform within and between lifecycle phases in case of PLM from existing literature.

Long term and short term benefits and risks of platform openness related component This component brings out the long term and short term benefits and risks of platform openness for platform end-users that might effect the selection of industrial internet platforms for the end-users. Section 2.3 brings out certain long and short term benefits and risks of platform openness for platform end-users from the literature.

\section{Results and Findings}

Based on the interviews conducted with Kaa-IoT and PTC ThingWorx personnel, we present the results and the findings in this section.

Strategy component For Kaa-IoT the overall strategy of their industrial internet platform is to move from a services company to a product centric company. Kaa IoT - "The services business model has restrictions, growth related. Hence Kaa will have an enterprise cloud which will make Kaa a PAAS (Platformas-a-service). Reason for this strategy is that it is easier to sell products than engineering hours." For PTC Thingworx, moving towards shared model from the existing licensing model using partnerships (with other industrial internet platforms) is the overall strategy. PTC ThingWorx - "Leverage partners to grow PTC's industrial internet business is the overall growth strategy". For Kaa-IoT Robotics and sophisticated as well as complex manufacturing industries are the 
target industries for their industrial internet platforms. PTC ThingWorx is focusing on Oil and Gas, Mining, Smart Cities, solutions related to tracking and logistics as part of their industrial internet strategy.

Table 2. Findings from Kaa IoT and PTC ThingWorx based on Openness dimensions

\begin{tabular}{|c|c|c|c|c|c|}
\hline \multirow[b]{2}{*}{ Openness Criteria } & \multirow[b]{2}{*}{ Detailed Criteria } & \multicolumn{2}{|c|}{$\begin{array}{c}\text { Investment Priority (green color) } \\
\text { and level of openness } \\
\text { (Scale 1-not very } \\
\text { open 5- very open) }\end{array}$} & \multicolumn{2}{|c|}{$\begin{array}{l}\text { Details of } \\
\text { openness } \\
\text { for every } \\
\text { dimension }\end{array}$} \\
\hline & & Kaa IoT & PTC ThingWorx & Kaa IoT & PTC ThingWorx \\
\hline \multirow[t]{3}{*}{$\begin{array}{l}\text { Demand-side user } \\
\text { openness } \\
\text { (Platform User) }\end{array}$} & $\begin{array}{l}\text { Access to } \\
\text { information } \\
\text { (Openness } \\
\text { standards) }\end{array}$ & \multirow{3}{*}{-1} & \multirow[t]{3}{*}{5} & $\begin{array}{l}\text { Apache } 2.0 \text { protocols, } \\
\text { various latest industry } \\
\text { protocols, security } \\
\text { standards }\end{array}$ & $\begin{array}{l}\text { Java application } \\
\text { working on a } \\
\text { browser, Apache } \\
\text { libraries, Connectivity } \\
\text { agnostic, works as } \\
\text { middleware }\end{array}$ \\
\hline & \multirow{2}{*}{\begin{tabular}{|l|} 
Cost of access \\
$\begin{array}{l}\text { Control in terms } \\
\text { of rules to } \\
\text { use the platform }\end{array}$
\end{tabular}} & & & No cost of access & $\begin{array}{l}\text { Three payment } \\
\text { models: Standard, } \\
\text { Enterprise and } \\
\text { Professional } \\
\end{array}$ \\
\hline & & & & User decides the rules & User decides the rules \\
\hline \multirow{3}{*}{$\begin{array}{l}\text { Supply-side user } \\
\text { openness } \\
\text { (Application } \\
\text { Developer) }\end{array}$} & Core developers & \multirow{3}{*}{$\begin{array}{l}5 \text { (Application } \\
\text { developers } \\
\text { are real end-users } \\
\text { of Kaa IoT) }\end{array}$} & \multirow{3}{*}{$\begin{array}{l}4 \text { (For PTC } \\
\text { ThingWorx,plat- } \\
\text { form is an applic- } \\
\text { ation } \\
\text { developer tool) }\end{array}$} & There is no difference & No core developers \\
\hline & \begin{tabular}{|l|} 
Extension \\
(3rd Party) \\
developers \\
\end{tabular} & & & $\begin{array}{l}\text { in terms of access } \\
\text { to data for core or } \\
\text { 3rd party developers. }\end{array}$ & $\begin{array}{l}\text { for application } \\
\text { development, applications } \\
\text { are made only by }\end{array}$ \\
\hline & \begin{tabular}{|l|} 
Data \\
aggregators
\end{tabular} & & & $\begin{array}{l}\text { Data aggregation } \\
\text { is not allowed }\end{array}$ & $\begin{array}{l}\text { 3rd-party developers. } \\
\text { Data aggregation is allowed }\end{array}$ \\
\hline $\begin{array}{l}\text { Platform provider } \\
\text { and sponsor } \\
\text { related openness }\end{array}$ & \begin{tabular}{|l|}
$\begin{array}{l}\text { Proprietary } \\
\text { model }\end{array}$ \\
$\begin{array}{l}\text { Licensing } \\
\text { model }\end{array}$ \\
$\begin{array}{l}\text { Joint venture } \\
\text { model }\end{array}$ \\
Shared model \\
\end{tabular} & $\begin{array}{l}5 \text { (Shared } \\
\text { model-mostly } \\
\text { hardware } \\
\text { partners-no } \\
\text { platform } \\
\text { partners) }\end{array}$ & $\begin{array}{l}4 \text { (With more } \\
\text { platform partners } \\
\text { moving towards } \\
\text { shared model) }\end{array}$ & $\begin{array}{l}\text { Currently under } \\
\text { shared model } \\
\text { and would } \\
\text { continue } \\
\text { to be } \\
\text { shared model }\end{array}$ & $\begin{array}{l}\text { Currently in between } \\
\text { licensing and shared } \\
\text { model, } \\
\text { would } \\
\text { move to } \\
\text { shared model }\end{array}$ \\
\hline
\end{tabular}

Openness dimensions related component Table 2 summarizes the findings based on openness dimensions. Both Kaa-IoT and PTC Thingworx consider the supply-side user openness (marked in green in Table 2.) dimension as the priority when it comes to investment out of the three dimensions. Kaa-IoT "Application developers are the real end user of the platform. Application developers should develop unique and useful applications for industries to use and make value from." Whereas, for PTC Thingworx, "Platform is an application developer tool", i.e. even if the industrial customers are the real end users of the platform, applications contribute maximum to the value creation. When asked to rate from 1-5 (1-not very open and 5-very open) the three dimensions, KaaIoT rated all the dimensions as 5. PTC ThingWorx rated the dimensions as 5, 4 and 4 . For the access to information sub-dimension, Kaa-IoT uses Apache 2.0 protocol (the most open protocol), latest industry protocols (related to interoperability) and security standards. PTC ThingWorx is a java application working on any browser, uses apache libraries, connectivity agnostic and also can work as a middleware with wrappers around. In case of the cost of access dimension, Kaa IoT is completely free at all stages and for all kinds of industries whereas PTC ThingWorx has three payment models, Standard, Enterprise and Professional 
models. For the control in terms of rules to use the platform, for both the platforms, users decide the rules. As far as the application developers are concerned, for Kaa-IoT there is no difference in the access to data between core developers and 3rd party developers. Kaa-IoT does not allow Data Aggregators to access the platform. Whereas for PTC ThingWorx, they dont have core developers for application development, applications are made only by 3rd party developers and there is no restriction with respect to data for them. Data aggregation is allowed but the control is given to the end-user. For the final dimensions both the platforms have similar strategy of being very open by adopting sharing model but the difference lies in the kind of partnerships for both the platforms. Kaa-IoT is not going to partner with any other industrial internet platform. Partnership with complementors, hardware suppliers is what drives Kaa-IoT's sharing model. For PTC ThingWorx, its very different. All kinds of partnerships, i.e., with competing industrial internet platforms, hardware suppliers, system integrators etc is the key driving force for their sharing model.

PLM component As depicted in Table 3, the focal IIoT platform providers have different ambitions when it comes to the different product lifecycle phases. PTC's strategic goals align with the potential offered by their sophisticated and comprehensive set of legacy systems spanning all phases of the lifecycle (design to disposing). Kaa, as an open source IIoT platform provider, allows and encourages end-users to customize their system to a large extent, which brings forth the possibility of solutions for the different lifecycle phases. However, they are not natively build in and/or connected to the platform itself. Most of the customers of Kaa use the platform for managing their MOL and EOL phases. This focus on MOL/EOL seems to stand true for most IIoT platforms today, while some start to include the later part of the BOL, manufacturing, assembly and logistics, in their core offering. With regard to full BOL support and in-

Table 3. Usage of industrial internet platforms within \& between lifecycle phases

\begin{tabular}{|l|l|l|l|}
\hline & BOL & MOL & EOL \\
\hline PTC-ThingWorx & & & \\
\hline Kaa-IoT & & & \\
\hline
\end{tabular}

clusion, PTC stands out within the IIoT platform landscape due to their own PTC CREO solution. This access to an product design solution allows them to provide full support for closed-loop lifecycle management. With such a comprehensive coverage, the previously theorized option of incorporating MOL/EOL (IoT) data directly in the design of the next generation of products is coming within reach for industry/manufacturers. This functionality is also possible with other platforms, e.g., Kaa's, through developing the required apps and interoperability standards/interfaces, and thus providing the MOL/EOL data to the CAD solution (BOL).

Long term and short term benefits and risks of platform openness related component Table 4 shows the platform end-user or platform customer short and long term risks and benefits of platform openness from the platform provider's perspective. For short term benefits category Kaa-IoT considers the 
platform to be production and implementation ready. The platform is completely free, so for smaller companies it will save costs. Being open source, Kaa IoT allows any developer to develop applications. For PTC ThingWorx, the short term benefits include the fact that platform is ready to use and has a very low entry barrier for the end-user. Although the platform is not completely free but

Table 4. Short \& long term - benefits \& risks of platform openness for end-users

\begin{tabular}{|c|c|c|c|c|}
\hline & \multicolumn{2}{|c|}{$\begin{array}{l}\text { Short Term } \\
\end{array}$} & \multicolumn{2}{|c|}{ Long Term } \\
\hline & $\overline{K a a-I o T}$ & PTC-ThingWorx & Kaa-IoT & PTC-ThingWorx \\
\hline Benefits & $\begin{array}{l}\text {-Being free, for small } \\
\text { companies it would } \\
\text { save costs }\end{array}$ & $\begin{array}{l}\text {-Platform users have a low } \\
\text { barrier to entry. Platform } \\
\text { is ready to use. } \\
\text {-Not completely free, } \\
\text { but with small internal } \\
\text { resources companies } \\
\text { can start development } \\
\text { quickly, without any } \\
\text { technological investments }\end{array}$ & $\begin{array}{l}\text {-Larger pool of engineers } \\
\text { would be available } \\
\text { to custom make } \\
\text { the solutions for industries } \\
\text {-Because it was customized by } \\
\text { the end-user, end-user } \\
\text { becomes an advanced } \\
\text { expert in the technology. } \\
\text { With industrial internet } \\
\text { becoming part of } \\
\text { the core business, this } \\
\text { is an advantage } \\
\text {-As the end-user matures, } \\
\text { it will have many devices } \\
\text { connected to the platform, } \\
\text { and because the platform is free, } \\
\text { end-user company will } \\
\text { save lot of costs }\end{array}$ & $\begin{array}{l}\text {-Platform would allow } \\
\text { people to join you } \\
\text { later - work with } \\
\text { partners, 3rd parties, } \\
\text { even competitors etc } \\
\text {-ThingWorx is a widely } \\
\text { used platform, considering } \\
\text { the number of companies } \\
\text { and the revenue, which } \\
\text { means in long term it will } \\
\text { be more sustainable to use } \\
\text { ThingWorx as a platform } \\
\end{array}$ \\
\hline Risks & $\begin{array}{l}\text {-Platform is complicated } \\
\text { and complex, hence } \\
\text { it is not a plug-n-play } \\
\text { kind of platform } \\
\text {-Platform requires sophis- } \\
\text { ticated programming } \\
\text { for implementation } \\
\text {-Platform requires to be } \\
\text { customized by the } \\
\text { end-user } \\
\text { in order to use it in their business }\end{array}$ & $\begin{array}{l}\text {-Requires maturity and } \\
\text { experience in the } \\
\text { end-user organization }\end{array}$ & $\begin{array}{l}\text {-Platform is open source and } \\
\text { if the open source } \\
\text { community stops } \\
\text { the development then } \\
\text { the platform will cease to exist } \\
\text {-Lack of a big investor/sponsor } \\
\text { might effect the future } \\
\text { development of the platform }\end{array}$ & $\begin{array}{l}\text {-Data management related } \\
\text { risks, for example, data } \\
\text { storage, data integrity }\end{array}$ \\
\hline
\end{tabular}

with very low internal resources the end-users can start development without any other technological investments. In case of long term benefits for Kaa-IoT, their strategy is centered around open source model. Because of being open source, over time, large pool of engineers would be available to custom make solutions for the industries using the capabilities of Kaa IoT. The platform end-user or the customer was the one who customized and engineered the platforms as per their company's requirement. Hence, over years the end-user becomes an expert with the technology that they developed using Kaa-IoT. With industrial internet becoming the central focus of all future business models, this is a big advantage. As the end-user matures and deploys many more devices that are now connected using Kaa-IoT saves a lot of costs, because Kaa-IoT would be still free for them. For PTC Thingworx, the long term benefits include the fact that the platform allows other firms, 3rd parties, even competitors to join in the en-user's platform instance at any given point of time. Currently, PTC ThingWorx is one of the most widely used platforms in terms of user base and revenue generated. This makes PTC Thingworx a very sustainable platform as the time progresses. Short term 
risks for Kaa-IoT include the fact that the platform is complicated and complex, hence it requires implementation skills. It is not a plug-n-play kind of platform like other commercial platforms. Kaa-IoT requires sophisticated programming skills to implement the platform before the end-user can actually use it. Platform needs to be customized by the end-user as per their business requirements. In case of PTC ThingWorx, short term risks include lack of maturity and experience from the end-user leading to under-usage of the platform initially. Other short term risks for PTC thingworx are scalability and security risks. In case of long term risks for Kaa-IoT, it being open source, it is heavily reliant on the open source community to further develop in the future or else it would cease to exist. Lack of a big investor/sponsor might also effect the future development of KaaIoT which would effect the end-user in long term. In case of PTC Thingworx, long term risks are around security and data management related risks.

\section{$5 \quad$ Discussion and Conclusions}

Our purpose was to analyze strategy of platform provider's towards openness and related dimensions as well as short and long term benefits and risks of the aforementioned strategy.

For both the industrial internet platforms, Kaa-IoT and PTC ThingWorx, openness plays a very important role in their current as well future strategies related to the growth of the platform. Kaa-IoT, is primarily a services company where they provide engineering services around the implementation of the platform. They will become a more product oriented company by releasing their enterprise cloud built around the open source Kaa-IoT platform. This strategy will be useful for scaling up the business of Kaa-IoT. For PTC ThingWorx, openness, allows them create more partnerships with other industrial internet platforms like, Azure, GE Predix, Amazon AWS and others. Partnerships of these kind will allow them to grow the platform business using network effects as well as interoperability resulting from openness.

The above mentioned openness related overall strategy resulted in prioritizing the supply-side user openness dimension (as shown in Table 2) over the other two for both the platform companies for similar reasons. For both the platforms, application developers create the solutions that industrial end-users will create value and grow business. Hence, investing into developing applications is very important.

Although Kaa-IoT is free to use and open source, but being complex for implementation makes it difficult to adopt for industrial customers who are not mature in industrial internet technologies. PTC Thingworx, on the other hand, has a payment model for every kind of user group but implementing the platform is simple because of the plug-n-play mechanism (Table 4). In long term, the complex implementation of Kaa-IoT would actually be a benefit for industrial customers because they would be experts of their own unique technology platform which will lead to better security practices when compare to commercial platforms like PTC Thingworx. On the other hand if the open source community 
stops developing Kaa-IoT's platform technology, it would cease to exist and cause a long term risk for their industrial customers, unlike PTC ThingWorx, that has a big brand like PTC to sustain the development of the platform. PTC's historical expertise in PLM related issues allows ThingWorx to accommodate all the PLM challenges [16] effectively within and between lifecycle phases. KaaIoT's focus on MOL-EOL data, information and knowledge exchange creates a limitation for the industrial companies to facilitate the BOL phase, but being open source this can be solved by developing an additional module to the existing platform stack. Both options have their advantages and disadvantages, e.g., while PTC's comprehensive solution offers native integration, it also forces companies to constrain themselves to one provider. For supplier's which are required to work with the OEM's CAD system, this might present a problem. Other providers, who focus on MOL/EOL (IoT) data might not offer the native support of the whole product lifecycle, but due to that lack of BOL coverage, they might be forced to think more in terms of interoperability and offering interfaces to interact with BOL (CAD) solutions of other providers. In the end, it depends on the individual company and their strategic goal, customer/supplier base and existing infrastructure which approach might be more suited for their needs.

The studied platforms consider openness as high priority (Table 2) despite difference in their strategy and different positioning in the continuum of openness. This is also reflected in the manner that the platforms have rated (Very High) the various dimensions of openness in order to provide maximum value to industrial end-users. (RQ1)

Supply-side user (application developer) openness is a dimension where both companies plan to invest significantly. This helps to customize and add features as per customers needs which in turn enhances the value of the platform for the end-users. Both companies (PTC and Kaa) make use of enhanced openness (in some respect) in order to improve the data, information and knowledge exchange within and between the lifecycle phases. Industrial internet platforms allow to close the loop in the true meaning of the word with respect to data information and knowledge in case of PLM. (RQ2)

Both the platforms explicated different types of both short and long terms impacts of openness (as in Table 4) which were related mainly to quick and low barrier implementation of platforms as well as cost of usage and access ( low cost on investment means the platform can be easily tested and experimented by SME's). (RQ3)

The purpose of this study was also to allow managers of platforms and industrial end-users to get an insight into the strategy of industrial internet platforms like Kaa-IoT and PTC ThingWorx. It allows them to select a platform based on their needs as well as keeping in mind the long term and short term benefits as well as risks of industrial internet platform selection. The limitation of this study was the limited number of industrial internet platform cases that were presented. The future studies will include other leading industrial internet plat- 
forms as well as industrial end-user viewpoints on the issue of different types of openness and their impacts to the business.

\section{References}

1. Hermann, M., Pentek, T., Otto, B.: Design Principles for Industrie 4.0 Scenarios. In: 2016 49th Hawaii International Conference on System Sciences (HICSS), IEEE (jan 2016) 3928-3937

2. Lee, I., Lee, K.: The Internet of Things (IoT): Applications, investments, and challenges for enterprises. Business Horizons 58(4) (jul 2015) 431-440

3. Evans, P.C., Annunziata, M.: Industrial internet: Pushing the boundaries of minds and machines. (2012)

4. Iansiti, M., Lakhani, K.: Digital Ubiquity. How Connections, Sensors, and Data Are Revolutionizing Business. Harvard Business Review 92(11) (2014) 90-99

5. Porter, M.E., Heppelmann, J.: Managing the Internet of Things : How Smart , Connected Products are Changing the Competitive Landscape. Harvard Business Review (2014) 2014-2016

6. Kiritsis, D.: Closed-loop PLM for intelligent products in the era of the Internet of things. Computer-Aided Design 43(5) (2011) 479-501

7. Gawer, A., Cusumano, M.A.: Industry platforms and ecosystem innovation. Journal of Product Innovation Management 31(3) (may 2014) 417-433

8. Golovatchev, J., Chatterjee, P., Kraus, F., ...: The Impact of the IoT on Product Development and Management. ISPIM Innovation ... (2016)

9. Jun, H.B., Kiritsis, D., Xirouchakis, P.: Research issues on closed-loop PLM. Computers in Industry 58(8-9) (2007) 855-868

10. Schreieck, M.: Design and Governance of Platform Ecosystems - Key Concepts and Issues for Future Research. In: ECIS 2016. (2016)

11. Eisenmann, T.R.: Managing Proprietary and Shared Platforms. California Management Review 50(4) (2008)

12. Agarwal, N., Brem, A.: Strategic business transformation through technology convergence: implications from General Electric's industrial internet initiative. International Journal of Technology Management 67(2-4) (jan 2015) 196-214

13. Heppelmann, J., Porter, M.E., Heppelmann, J.E.: How Smart, Connected Products Are Transforming Competition. Harvard Business Review 92(11) (2014)

14. Porter, M.E.: HOW SMART, CONNECTED PRODUCTS ARE TRANSFORMING COMPANIES The. HBR 1 (2015)

15. Terzi, S., Bouras, A., Dutta, D., Garetti, M., Kiritsis, D.: Product lifecycle management - from its history to its new role. International Journal of Product Lifecycle Management 4(4) (2010) 360

16. Menon, K., Kärkkäinen, H., Gupta, J.P.: Role of Industrial Internet platforms in the management of product lifecycle related information and knowledge. IFIP Advances in Information and Communication Technology. Springer Berlin Heidelberg (jul 2016)

17. Parker, G.G., Van Alstyne, M.W., Choudary, S.P.: Platform revolution: How Networked Markets Are Transforming the Economy-And How to Make Them Work for You. (2016)

18. Eisenmann, T.R., Parker, G., Van Alstyne, M.: Opening Platforms: How, When and Why? In: Platforms, Markets and Innovation. (2009) 131-162 
19. Wuest, T., Wellsandt, S., Thoben, K.D.: Information Quality in PLM: A Production Process Perspective. Springer International Publishing (2016) 826-834

20. Wellsandt, S., Nabati, E., Wuest, T., Hribernik, K., Thoben, K.D.: A survey of product lifecycle models: towards complex products and service offers. International Journal of Product Lifecycle Management 9(4) (2016) 353-390

21. Lehmhus, D., Wuest, T., Wellsandt, S., Bosse, S., Kaihara, T., Thoben, K.D., Busse, M.: Cloud-Based Automated Design and Additive Manufacturing: A Usage Data-Enabled Paradigm Shift. Sensors 15(12) (dec 2015) 32079-32122

22. Eriksson, P., Kovalainen, A.: Qualitative Methods in Business Research. Oxford University Press 3 (2008) 675 